\title{
Zoneamento Geoambiental e Unidades da Paisagem: os casos dos estados de Ceará e Rio Grande do Norte
}

\author{
Geoenvironmental Zoning and Units of Landscape: the cases of the states of Ceará and Rio \\ Grande do Norte
}

\author{
Guedes $^{1}$, D. R.C.; Diniz ${ }^{2}$, M. T. M.; Lima 33 , Z. M. C; Silva ${ }^{4}$, S. D. R.; Araújo ${ }^{5}$, I. S \\ dayane.geo10@gmail.com;
}

\section{Resumo}

O presente artigo tem como objetivo analisar a importância dos zoneamentos geoambientais para os estudos integrados da paisagem, considerando categorias de análise indispensáveis a tais estudos, como por exemplo, Geossistema e Paisagem. Para tal, fez-se uma ampla revisão de literatura pautada não só na questão central, como também em temáticas relacionadas aos estudos de unidades da paisagem. Esta discussão, em torno do conceito de zoneamento, possibilita a compreensão aprofundada de como a interface sociedadenatureza é indispensável para a apreensão do conceito de Espaço Geográfico, que é central na Geografia, além de permear estudos sistêmicos e eficientes do meio. Por fim, foi apresentada uma comparação entre propostas de zoneamentos já realizadas nos estados do Ceará e do Rio Grande do Norte.

Palavras-chave: Zoneamentos Geoambientais; Geossistema; Análise Integrada da Paisagem.

\section{Abstract}

This paper aims to analyze the importance of geoenvironmental zoning for the integrated study of the landscape, considering categories of analysis necessary for such studies, such as geosystem and landscape. To this end, became an extensive literature review based not only on the central issue, as well as in thematic related to landscape units of study. This discussion, around the concept of zoning, enables a depth understanding of how society-nature interface is essential for the apprehension of the concept of Geographic Space, which is central in geography, and pervade systemic and efficient environmental studies. Finally, was presented a comparison of proposed zonings already held in the states of Ceará and Rio Grande do Norte.

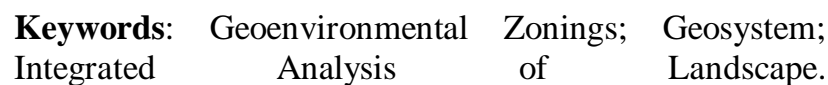

\section{INTRODUÇÃO}

A análise sistêmica surge como um suporte para o entendimento da interação dos elementos naturais e antrópicos. Deste modo, compreender a dinâmica e estrutura da paisagem torna-se importante para um uso dos recursos naturais em consenso com as suas potencialidades e limitações (LIMA, CESTARO e ARAÚJO, 2010).

${ }^{1}$ Guedes, Dayane Raquel da Cruz. Departamento de Geografia - Laboratório Multiusuário de Monitoramento Ambiental, Universidade Federal do Rio Grande do Norte, Caicó - RN, Brasil.

${ }^{2}$ Diniz, Marco Túlio Mendonça. Departamento de Geografia-Laboratório de Geoprocessamento e Geografia Física, Universidade Federal do Rio Grande do Norte, Caicó-RN, Brasil

${ }^{3}$ Lima, Zuleide Maria Carvalho. Departamento de Geografia - Laboratório de Geografia Física, Universidade Federal do Rio Grande do Norte, Natal - RN, Brasil.

${ }^{4}$ Silva, Sandro Damião Ribeiro da. Departamento de Geografia - Laboratório de Geografia Física, Universidade Federal do Rio Grande do Norte, Natal-RN, Brasil.

${ }^{5}$ Araújo, Isailma da Silva. Departamento de Geografia - Laboratório de Geografia Física, Universidade Federal do Rio Grande do Norte, Natal-RN, Brasil. 
Dessa forma, o Zoneamento Geoambiental passou a integrar a Geografia a partir do momento em que houve uma preocupação pela necessidade da preservação e da sustentabilidade ambiental, como também da melhor adequação de espaços sociais. A partir deste momento, zoneamento e planejamento ambiental passaram a caminhar lado a lado (SANTOS, 2004; PILACHEVSKY, 2013).

Para Zacharias (2006), por sua vez, a utilização de zoneamento geoambiental possibilita a identificação de áreas suscetíveis à ocorrência de processos do meio físico e a avaliação das áreas quanto ao seu potencial para o desenvolvimento de atividades antrópicas, visando facilitar o planejamento, por exemplo.

Baseado nestas perspectivas, o presente artigo tem como objetivo discorrer, teoricamente, sobre zoneamento geoambiental e unidades da paisagem, trazendo os exemplos de mapeamentos dos estados do Ceará e do Rio Grande do Norte, comparando-os e apontando falhas e acertos em cada um deles.

\section{METODOLOGIA}

Os procedimentos metodológicos da pesquisa tiveram início com uma intensa revisão bibliográfica, na qual autores que trabalham com a ideia de zoneamento (numa perspectiva mais geral e em casos mais específicos, também) foram postos em evidência. Tal revisão procurou corroborar o sistema taxonômico proposto por Georges Bertrand (1972), o qual serviu de subsídios para os estudos de Marcos Souza (2000) e Luiz Cestaro et al (2006). No tocante à análise Ecodinâmica de Jean Tricart (1977), que não fora deixada de lado nessa pesquisa, foi consultado Souza (2000) como principal referência no que tange a tal análise em áreas semiáridas brasileiras.

Além disso, foi tecida uma análise comparativa acerca dos zoneamentos propostos, de modo que leituras acerca das propostas também foram executadas, afim de se buscar evidências de correções e aperfeiçoamentos propostas nos últimos anos. Para tal, os critérios definidos para a elaboração das compartimentações potiguar e cearense foram buscados e retrabalhados.

\section{RESULTADOS E DISCUSSÃO}

\subsection{Zoneamento Geoambiental}

Zoneamento é a compartimentação de uma região em porções territoriais, obtida pela avaliação dos atributos mais relevantes e de suas dinâmicas. A análise é feita através das potencialidades, fragilidades, suscetibilidade, acertos e conflitos de um território (SANTOS, 2004; 
ZACHARIAS, 2006). Para Almeida e Soares (2009), o zoneamento é configurado como um instrumento de ordenamento territorial, que resultará num manejo da unidade, estabelecendo usos diferenciados para cada zona, de acordo com seus objetivos.

Independentemente dos adjetivos a que estão associados, os tipos de zoneamento possuem um resultado em comum, que são a definição de zonas, mas sua concepção pode ser bastante diferenciada, o que induz caminhos metodológicos bem distintos entre si, seja em função do objetivo, seja em função do objeto (SILVA E SANTOS, 2004).

O zoneamento tem um papel fundamental no planejamento ambiental, onde é uma estratégia metodológica que representa uma etapa do planejamento. No que diz respeito aos aspectos legais, para a Lei 6.938/81, que dispõe sobre a Política Nacional do Meio Ambiente, o zoneamento ambiental é considerado como uma integração sistemática interdisciplinar da análise ambiental ao planejamento do uso do solo, com objetivo de definir a gestão dos recursos ambientais. Ela foi a primeira legislação de importância para a inserção do zoneamento ambiental como instrumento de planejamento do meio físico, sendo regulamentada em 2002 pelo decreto $\mathrm{n}^{\circ}$ 4.297, com a denominação de Zoneamento Ecológico - Econômico (ZEE), (PILACHEVSKY, 2013).

O zoneamento geoambiental parte da visão sistêmica e se baseia, metodologicamente, no conceito de Ecodinâmica de Tricart (1977), que considera, em suas análises, as relações mútuas entre os diferentes componentes da dinâmica e os fluxos de energia e matéria (entrada e saída/input and output) no meio ambiente.

Segundo Pilachevsky (2013) e Silva e Santos (2004), o zoneamento geoambiental pode ser considerado um instrumento inter e multidisciplinar de planejamento. Assim, ele deve ter como meta o fornecimento de subsídios técnicos para orientar e elucidar a tomada de decisões na implementação de alternativas de desenvolvimento regional, compatíveis com a sustentabilidade e vulnerabilidade dos sistemas ambientais (OHARA, et al, 2003). Nesse sentindo, o zoneamento geoambiental consiste no resultado da análise integrativa dos elementos que compõem uma paisagem, sendo, destarte, de fundamental importância para o planejamento do território.

\subsection{Unidades da Paisagem}

De um modo geral, os zoneamentos consideram subdivisões, que além de irem ao encontro do tipo de escala manejado, mostram que algumas unidades taxonômicas podem apresentar um grau de relevância variado em relação ao que se pretende mostrar. Isso é permeado no âmbito da análise física integrada da paisagem, cujo estudo busca enfocar todos, ou pelo menos a maioria, dos aspectos geográficos de um dado espaço. Nesse contexto, destaca-se a Teoria Geral dos Sistemas 
(TGS), proposta pelo austríaco Ludwig Von Bertalanffy (1968), na qual os sistemas podem ser considerados em diversas escalas de abrangência geográfica e ainda podem ser divididos em subsistemas ou subclasses.

Após a TGS, estudiosos europeus desenvolveram trabalhos de referência na perspectiva do estudo integrativo da Paisagem, conceito este que ganhava cada vez mais força no âmbito da ciência geográfica. Entre eles, podem-se mencionar os estudos de geossistemas de Vitor Sotchava (1977), de paisagem (num contexto global) de Georges Bertrand (1972) e da Ecodinâmica de Jean Tricart (1977). Baseado nisto, Santos Júnior (2013) salienta a TGS como divisor de águas entre estudos mecanicistas da paisagem e a eficácia de estudos sistêmicos, comprovada nos dias atuais.

A ideia de Geossistema, por sua vez, é uma das mais difundidas na Geografia. Tal ideia surgiu com Sotchava (1977) na extinta União Sovietica. Para o russo, os geossistemas podem refletir parâmetros sociais e econômicos (CHRISTOFOLETTI, 1999), que influenciam importantes conexões em seu interior. Bertrand, por sua vez, propôs um sistema taxonômico de classificação da paisagem, a saber: Zona, Domínio e Região Natural (unidades superiores); Geossistema, Geofácie e Geótopo (unidades inferiores).

O geossistema deu à Geografia Física melhor caráter metodológico, facilitando e incentivando os estudos integrados das paisagens. Desta forma, pode-se afirmar que o geossistema colaborou para as análises ambientais em Geografia, pois possibilita um prático estudo do espaço geográfico com a incorporação da ação social na interação natural com o potencial ecológico e a exploração biológica (NASCIMENTO e SAMPAIO, 2004).

Entretanto, como bem afirmam Diniz, Oliveira e Medeiros (2015), a ideia de Geossistema está muito acima de uma simples unidade taxonômica, estando mais próxima de uma categoria de análise dos estudos geográficos. Dessa maneira, tais autores ressaltam a noção de Geocomplexo, proposta pelo próprio Bertrand, como mais apropriada para a classificação taxonômica de paisagens. Assim, o Geocomplexo passou a ser uma escala de análise geográfica e o Geossistema o próprio conceito base de sua teoria, ou seja, dos estudos da geografia física global. O estudo do Geocomplexo, aliás, é muito pertinente, pois este nível de escala (4 ${ }^{\text {a }}$ grandeza na escala de Bertrand) consegue "evidenciar as combinações dialéticas mais interessantes para o geógrafo compatíveis com a escala humana" (DINIZ, OLIVEIRA e MEDEIROS, 2015, p. 55).

\subsection{Aplicação de Zoneamentos no Ceará e no Rio Grande do Norte}

\subsubsection{Proposta de classificação de paisagens no Rio Grande do Norte}


No contexto potiguar, Cestaro et al (2006) propuseram para o Rio Grande do Norte um sistema de classificação das unidades da paisagem que servisse de base para zoneamentos geoambientais futuros. O estado foi compartimentando em 9 (nove) Regiões Naturais, 17 (dezessete) Geossistemas (ou Geocomplexos) e 35 (trinta e cinco) Geofácies. As Regiões Naturais, são: Depressão Sertaneja, Planalto da Borborema, Planalto Residual, Morros Basálticos Isolados, Tabuleiro, Chapada, Planície Costeira, Planície Flúvio-marinha e Planície Fluvial e Lagunar.

Nesse trabalho, as unidades geoambientais foram classificadas a partir das categorias taxonômicas proposta por Bertrand (1972) e hierarquizados em ordem de Região Natural, Geossistema e Geofácie. E os parâmetros geológicos, geomorfológicos, climáticos, pedológicos e bióticos (vegetacionais), foram utilizados na compartimentação ambiental (CESTARO et al, 2006).

Assim, esta classificação (Figura 1) pode ser considerada como a primeira aproximação que se fez na tentativa de, utilizando uma abordagem geossistêmica e integradora, compartimentar o ambiente natural do Rio Grande do Norte em unidades geoambientais. 


\section{RIO GRANDE DO NORTE}

\section{MAPA DO GEOSSISTEMA}

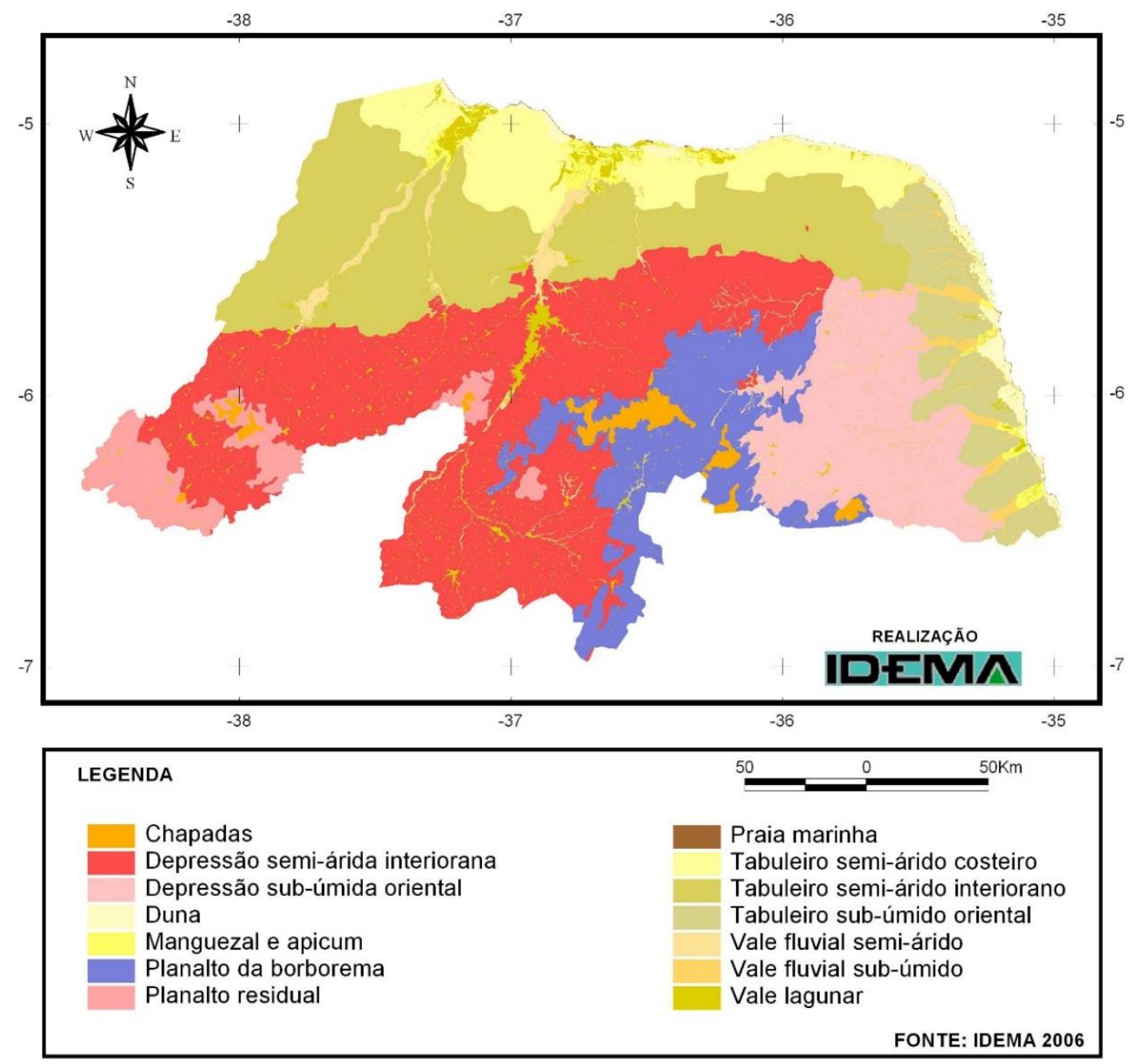

Figura 1: Mapa do Geossistema do Rio Grande do Norte. Fonte: Cestaro et al (2006).

\subsubsection{Proposta de classificação de paisagens no Ceará}

No caso específico do Ceará, por sua vez, Souza (2000) elaborou um mapeamento dos sistemas ambientais do estado do Ceará, em um sistema de hierarquização das paisagens semelhante ao elaborado por Cestaro et al (2006) para o Rio Grande do Norte. Esta obra, que tem por base a geomorfologia, tem servido de referência para diversos autores do estado cearense e de estados vizinhos. Ela tem servido, outrossim, para os órgãos e programas oficiais de planejamento do estado do Ceará como o Programa de Acão Estadual de Combate a Desertificação e Mitigação dos Efeitos da Seca que reproduziu a última versão do mapeamento de Souza para o Ceará (CEARÁ, 2009). Este zoneamento tem como base a escala de 1/500.000 e é fragmentado em 5 (cinco) domínios naturais, 13 (treze) sistemas ambientais e 32 (trinta e dois) subsistemas ambientais. 
O trabalho de Souza (2000), (Figura 2) também considerou as unidades geoambientais de acordo com a classificação taxonômica de Bertrand, hierarquizando-a em Domínios Naturais (Regiões Naturais), Sistemas Ambientais (Geocomplexos) e Subsistemas Ambientais (Geofácies). Ultrapassando as fronteiras cearenses, este trabalho é considerado o mais completo do gênero na Região Nordeste.

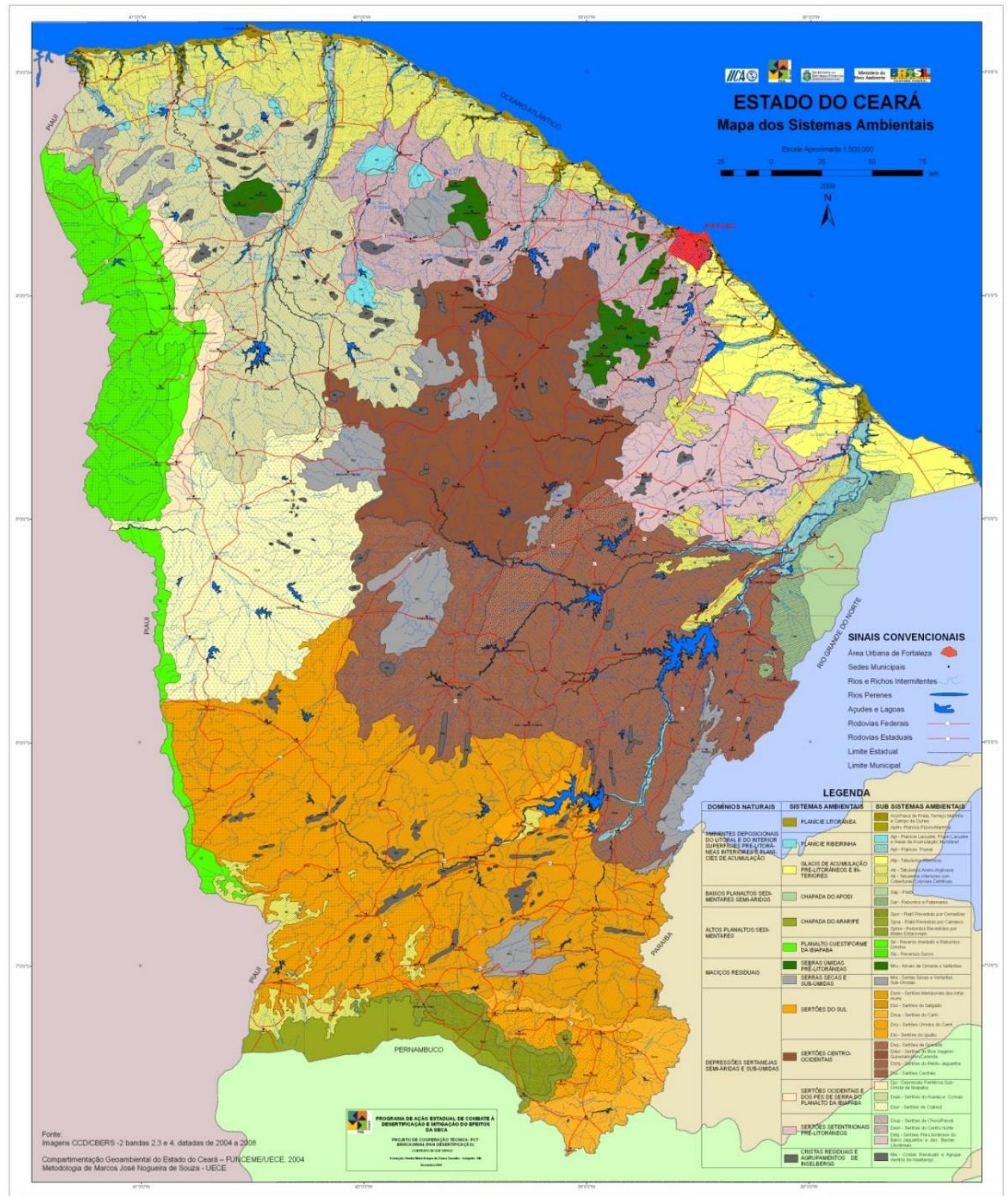

Figura 2: Mapa dos sistemas ambientais do Ceará. Fonte: Souza (2000).

3.3.3 Comparação entre as propostas de classificação de paisagens do Rio Grande do Norte e do Ceará 
É importante salientar que os mapeamentos de Cestaro et al (2006) e Souza (2000) são marcos consideráveis no âmbito da cartografia dos estados do Rio Grande do Norte e do Ceará, respectivamente. Ressalta-se, também, que ambos sofrerão, sem sombra de dúvidas, críticas, elogios e aperfeiçoamentos com o decorrer do tempo e do avanço de propostas cartográficas nos referidos estados. Todavia, aqui procuramos apontar algumas diferenças entre os dois, como forma de subsidiar revisões futuras ou que já estejam em andamento.

No caso do mapeamento do Rio Grande do Norte, Cestaro et al (2006) consideraram o parâmetro da vegetação como preponderante em sua análise sistêmica. Tal parâmetro também foi considerado por Bertrand em sua classificação taxonômica já abordada anteriormente. Aliás, na classificação taxonômica, o estudo potiguar traz o Geossistema como uma unidade taxonômica ${ }^{1}$.

Souza (2000), por sua vez, utilizou o caráter geomorfológico do Ceará como primordial no seu estudo, argumentando que, em áreas semiáridas nordestinas, onde o desmatamento é intenso, o relevo se apresenta como mais estável nas paisagens. Para tal, ele se baseia nas diversas obras do professor Aziz Nacib, como a divisão do Brasil em Domínios Morfoclimáticos (AB'SABER, 2003). Isso se constitui num grande acerto do autor cearense, uma vez que Bertrand, em sua classificação e em estudos posteriores, apontou para a necessidade de aperfeiçoamento do critério vegetacional em áreas com inúmeras particularidades ambientais, como é o caso do semiárido nordestino.Outro grande apontamento que se faz acerca do mapeamento de Souza (2000) é o seu caráter Ecodinâmico, extremamente atrelado à concepção geossistêmica de Sotchava e à taxonomia da paisagem de Bertrand. Isso está menos intensamente implícito no estudo de Cestaro et al (2006).

Ademais, na proposta de zoneamento potiguar, é possível notar que algumas unidades se encontram aparentemente superestimadas - como o geossistema da Depressão Semiárida Interiorana, o qual sugere-se ser subdividida. Outras áreas necessitam de agrupamento, como no caso do Geossistema Manguezal e Apicum. Aqui, entende-se que nesse nível de subdivisão estariam os geofácies. Contudo, cabe uma ressalva aqui: o zoneamento de Cestaro et al (2006) sofrerá alterações ao longo de anos, num processo contínuo de maturação, como o mapeamento de Souza (2000), que foi alvo de aperfeiçoamentos durante mais de uma década de pesquisas.

\section{CONSIDERAÇÕES FINAIS}

Os zoneamentos constituem-se como ferramentas indispensáveis à uma análise geográfica que preze pelo caráter sistêmico. Neles, é possível observar uma diversidade paisagística colossal

\footnotetext{
${ }^{1}$ É importante dizer que o esboço de zoneamento de Cestaro et al data de 2006. A revisão do conceito de Geossistema, passando a ser uma categoria de análise na ciência geográfica, por Bertrand, é posterior: 2007.
} 
que é, diariamente, estudada por geógrafos do mundo todo. Assim, os aspectos naturais podem servir de subsídios aos agentes sociais que permeiam o espaço geográfico.

Bertrand surge como aquele detentor de uma herança fascinante para a análise integrada da paisagem: o seu sistema taxonômico que propicia a fomentação de mapeamentos eficazes, cujos resultados possibilitam a construção de zoneamentos geoambientais, como os que aqui foram apresentados como eficientes no planejamento e na gestão territorial.

No caso específico desta pesquisa, enfatiza-se a maior complexidade do mapeamento de Souza (2000) para a geocartografia do Ceará. Alguns aspectos (consideração do Geossistema como categoria de análise; unidades geoambientais melhor delimitadas; e, o fator geomorfológico como elemento central na análise) tornam a sua análise uma referência para todo o Nordeste. Entretanto, o mapeamento de Cestaro et al (2006) apresenta um caráter inovador no âmbito da cartográfico do Rio Grande do Norte, não podendo deixar de ser elogiado por sua grandiosidade e importância, mesmo necessitando de aperfeiçoamentos.

Espera-se que, com essa análise dos mapeamentos de Souza (2000) e Cestaro et al (2006) e com os próprios estudos destes autores, novas propostas de aperfeiçoamento possa surgir ao longo do tempo, propiciando aquilo que prega o caráter teórico dos zoneamentos: o servir ao bem-estar social, a partir de análises sistêmicas e integradas da paisagem.

\section{REFERÊNCIAS}

AB'SÁBER, Aziz Nacib. Os Domínios de Natureza do Brasil: potencialidades paisagísticas. São Paulo, Ateliê Editorial, 2003.

ALMEIDA, F. G.; SOARES, L. A. A. Ordenamento territorial: coletânea de textos com diferentes abordagens no contexto brasileiro. Rio de Janeiro: Bertrand Brasil, 2009, 288p.

BERTALANFFY, Ludwig von. Teoria Geral dos Sistemas. Petrópolis, Vozes, 1968.

BERTRAND, Georges. Paisagem e Geografia Física global: esboço metodológico. Cruz, Olga (trad.) Cadernos de Ciências da Terra. São Paulo, USP-IGEOG, nº 43), 1972.

BRASIL. Lei no 6.938, de 31 de agosto de 1981.

CEARÁ, Secretaria dos Recursos Hídricos, Programa de Ação Estadual de Combate à Desertificação e Mitigação dos Efeitos da Seca, PAE-CE. Estado do Ceará: Mapa dos sistemas ambientais. Fortaleza: Ministério do Meio Ambiente/Secretaria dos Recursos Hídricas, 2009. Mapa colorido.

CESTARO, L. A.; ARAÚJO, P. C.; MEDEIROS, C. N.; CISNEIROS, R.; ARAÚJO, L. P. Proposta de um sistema de unidade geoambientais para o Rio Grande do Norte. Anais. In: XII Simpósio Brasileiro de Geografia Física Aplicada, Anais. Natal, 2006. 
CHRISTOFOLETTI, Antonio. Modelagem de Sistemas Ambientais. São Paulo: Edgard Blucher, 1999.

DINIZ, M. T. M.; OLIVEIRA, G. P.; MEDEIROS, D. B. S. Proposta de classificação das paisagens integradas. REGNE, Caicó, v.1, n.1, p. 50 - 65, mai. 2015.

LIMA, F. J.; CESTARO, L. A.; ARAÚJO, P. C. Sistemas geoambientais do município do Crato/CE. Mercator, V. 9, N. 19, p. 129 a 142, 2010.

NASCIMENTO, F. R.; SAMPAIO, J. L. F. Geografia física, geossistemas e estudos integrados da paisagem. Revista Casa da Geografia de Sobral, v. 06, n. 01, 2004.

OHARA, T.; JIMÉNEZ- RUEDA, J.R; MATTOS, J.T.; CAETANO, N. R. Zoneamento Geoambiental da região do Alto-Médio Rio Paraíba do Sul e a carta de aptidão física para a implantação de obras viárias. Revista Brasileira de Geociências, v.33, n. 2, p. 173-182, 2003.

PILACHEVSKY, T. Zoneamento geoambiental do município de São João da Boa Vista (SP). Rio Claro, 2013. Dissertação (Mestrado). Instituto de Geociências e Ciências Exatas, Universidade Estadual Paulista.

SANTOS JÚNIOR, R. R. D. A influência do Lago Artificial do Castanhão no Sistema Climático do Vale do Rio Jaguaribe-CE. Dissertação (Mestrado). Programa de Pós-Graduação em Geografia, Universidade Estadual do Ceará, 2013.

SANTOS, R. F. Planejamento Ambiental: teoria e prática. São Paulo: Oficina de Textos, 2004.

SILVA, J. S. V.; SANTOS, R. F. Zoneamento para planejamento ambiental: vantagens e restrições de métodos e técnicas. Cadernos de Ciência \& Tecnologia, v. 21, n. 2, p.221-263, 2004.

SOTCHAVA, V. B. O estudo dos geossistemas. Métodos em questão. n. 16, IGEOG-USP, São Paulo, 1977.

SOUZA, M. J. N. de. Bases Naturais e Esboço do Zoneamento Geoambiental do Estado do Ceará. In: LIMA, L. C., SOUZA, M. J. N. de, MORAIS, J. O. de. Compartimentação Territorial e Gestão Regional do Ceará. Fortaleza, Funece, 2000.

TRICART, J. Príncipes et Méthodes de la Geomorphologie. Paris, Ed. Masson et Cie, 1977.

ZACHARIAS, A. A. A representação gráfica das unidades de paisagem no zoneamento ambiental: um estudo de caso no Município de Ourinhos-SP. Rio Claro, 2006. Tese (Doutorado). Programa de Pós-Graduação em Geografia, Universidade Estadual Paulista.

\section{AGRADECIMENTOS}

Os agradecimentos desta pesquisa são canalizados para o Departamento de Geografia da Universidade Federal do Rio Grande do Norte (campus Natal e campus Caicó), os quais viabilizaram os Laboratórios de Geografia Física (LABGEOFIS), Multiusuário e Monitoramento Ambiental (LAMA) e de Geoprocessamento e Geografia Física (LAGGEF). Ambos se constituem nas bases diárias de pesquisa do grupo envolvido nessa pesquisa. 
Recebido em: 14/08/2016

Aceito para publicação em: 01/10/2016 